SUBJECT AREAS:

NANOPARTICLES

CHEMICAL SYNTHESIS

Received

14 October 2013

Accepted

4 February 2014

Published

17 March 2014

Correspondence and requests for materials should be addressed to B.Z.T. (tangbenz@ust. hk) or S.H. (sailing@ kth.se)

* These authors contributed equally to this work.

\section{Biocompatible and Photostable AIE Dots with Red Emission for In Vivo Two-Photon Bioimaging}

\author{
Dan Wang ${ }^{1,5 *}$, Jun Qian ${ }^{1 *}$, Wei Qin², Anjun Qin ${ }^{3}$, Ben Zhong Tang ${ }^{2,4} \&$ Sailing He${ }^{1}$
}

\begin{abstract}
${ }^{1}$ Centre for Optical and Electromagnetic Research, Zhejiang Provincial Key Laboratory for Sensing Technologies, Joint Research Center of Photonics of the Royal Institute of Technology and Zhejiang University (JORCEP), Zhejiang University, Hangzhou 310058 , China, ${ }^{2}$ Department of Chemistry, Division of Biomedical Engineering, and Institute for Advanced Study, The Hong Kong University of Science and Technology (HKUST), Clear Water Bay, Kowloon, Hong Kong, China, ${ }^{3}$ MoE Key Laboratory of Macromolecular Synthesis and Functionalization, Department of Polymer Science and Engineering, Zhejiang University, Hangzhou 310027 , China, ${ }^{4}$ Guangdong Innovative Research Team, SCUT-HKUST Joint Research Laboratory, State Key Laboratory of Luminescent Materials and Devices, South China University of Technology (SCUT), Guangzhou 510640, China, ${ }^{5}$ State Key Laboratory of OrganicInorganic Composites, Beijing University of Chemical Technology, Beijing 100029, China.
\end{abstract}

Bioimaging systems with cytocompatibility, photostability, red fluorescence, and optical nonlinearity are in great demand. Herein we report such a bioimaging system. Integration of tetraphenylethene $(T)$, triphenylamine $(T)$, and fumaronitrile $(\mathrm{F})$ units yielded adduct TTF with aggregation-induced emission (AIE). Nanodots of the AIE fluorogen with efficient red emission were fabricated by encapsulating TTF with phospholipid. The AIE dots enabled three-dimensional dynamic imaging with high resolution in blood vessels of mouse brain under two-photon excitation.

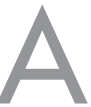

major thrust of biophotonics research is the development of fluorescent nanoparticle-based bioimaging systems $^{1}$. A wide variety of fluorescent materials have been used as probes or sensors for bioimaging applications, for example, organic dye-doped nanoparticles ${ }^{2,3}$, semiconductor quantum dots (QDs) ${ }^{4}$, metal nanodots ${ }^{5,6}$, and carbon nanomaterials ${ }^{7,8}$. Among these nanomaterials, organic dye-doped nanoparticles are promising candidates for clinical applications, because of their facile fabrication, low cytotoxicity, long-time circulation, and high photostability in physiological environment ${ }^{9,10}$. In the organic dye-doped nanoparticles, each particle can emit fluorescence of high intensity, due to the large number of dye molecules in the particle ${ }^{11}$. However, organic dyes tend to aggregate in the nanoparticles at high doping level. The dye aggregation often weakens or quenches light emissiona common phenomenon widely known as "aggregation-caused quenching" $(\mathrm{ACQ})^{12}$.

In 2001, Tang and coworkers observed an extraordinary phenomenon of "aggregation-induced emission" (AIE) in a silole fluorogen system ${ }^{13}$, which provides an ideal solution to the notorious ACQ problem ${ }^{14,15}$. The propeller-shaped silole molecules undergo dynamic intramolecular rotation in the solution state, which serves as a nonradiative relaxation path to annihilate their fluorescence process. However, when the fluorogen molecules form aggregates, the physical constraint in the aggregates restricts the intramolecular rotation (RIR) and blocks the nonradiative pathway. This opens the radiative decay channel, making the silole aggregates highly emissive. Under the guidance of the RIR mechanism, researchers have so far developed a large number of AIE fluorogen systems. AIE fluorogen-doped nanoparticles have been fabricated and found to work as bioprobes for in vitro cell imaging and in vivo sentinel lymph node mapping and tumor diagnosis ${ }^{16-21}$.

We have recently synthesized an AIE fluorogen called 2,3-bis(4-(phenyl(4-(1,2,2-triphenylvinyl)phenyl)amino)phenyl)-fumaronitrile (Fig. 1). The fluorogen is abbreviated as TTF, as it is an adduct of tetraphenylethene (T), triphenylamine (T), and fumaronitrile (F). In our previous work, we fabricated its nanoparticles functionalized by the cell-penetrating peptides derived from the transactivator of transcription proteins and used the resultant AIE dots as biotrackers for noninvasive long-term cell tracing ${ }^{22}$. We also prepared its nanoparticles using poly(lactideco-glycolide) as the encapsulation matrix and poly(vinyl alcohol) as the emulsifier through a solvent extraction/ evaporation procedure ${ }^{23}$. The polymer-encapsulated TTF nanoparticles functioned as bioprobes for in vitro cell imaging under one-photon excitation conditions ${ }^{23}$. 


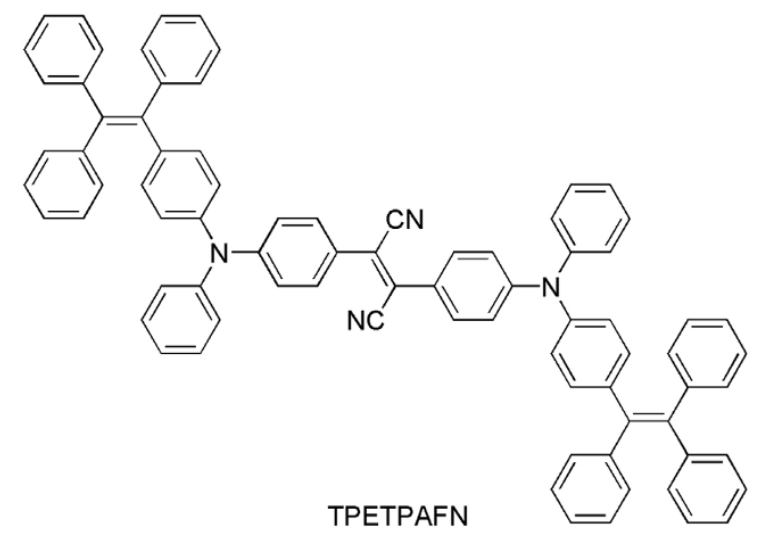

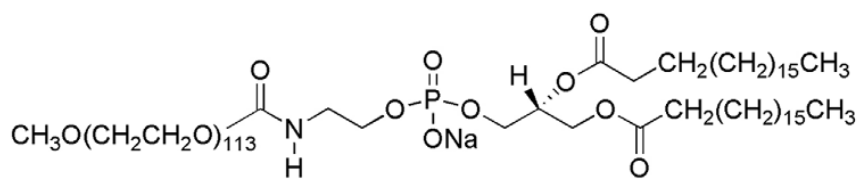

DSPE-mPEG 5000

Figure $1 \mid$ Chemical structures of the compounds forming AIE-dots in this study. TTF [an adduct of an adduct of tetraphenylethene (T), triphenylamine $(\mathrm{T})$, and fumaronitrile $(\mathrm{F})]$ and DSPE-mPEG 5000 [a poly(ethylene glycol) chain of 1,2-distearoyl-sn-glycero-3phosphoethanolamine-N-methoxy-(polyethylene glycol)-5000].

For in vivo bioimaging applications, the short-wavelength radiation used in the conventional one-photon systems can be easily absorbed by water in the biological tissues and scattered due to the Rayleigh scattering effect. As a consequence, the bioimaging depth becomes undesirably shallow. Two-photon laser scanning microscopy (TPLSM) is advantageous over its one-photon counterpart in terms of less scattering of the near-IR radiation, lower illumination power requirement, deeper penetration depth, and reduced phototoxicity. It is a powerful microscopic tool for three-dimensional (3D) in vivo imaging of biological tissues, particularly intravital vasculature systems (blood vessels, neuron networks in brain, etc. ${ }^{24,25}$.

The AIE dots of TTF are promising candidates as probes for twophoton microscopic in vivo bioimaging. Unlike inorganic QDs, the organic AIE dots do not "blink" and are compatible with living systems $^{22,23,26}$. Since water absorption of light in the 700-900 nm region in biological tissue is much lower than that of light in the visible light region, the red/near-IR emission properties (which the present AIE-dots also have), as well as the high fluorescence quantum yield, are beneficial to deep tissue imaging ${ }^{22,27}$. The AIE dots are also nonlinear optically active, showing a large two-photon absorption cross-section $^{22}$. In the AIE dots, the molecules of the TTF fluorogen are encapsulated by the molecules of a phospholipid carrying a poly(ethylene glycol) chain named 1,2-distearoyl-sn-glycero3-phosphoethanolamine-N-methoxy-(polyethylene glycol)-5000 (DSPE-mPEG ${ }_{5000}$; Fig. 1). The long PEG chains have the function of improving the long-time circulation of nanoparticles and helping the nanoparticles avoid capture/degradation by reticuloendothelial systems. Thus, such a nanostructure of AIE fluorogen encapsulated in phospholipid-PEG is beneficial for in vivo applications ${ }^{10,22,28-30}$.

In this work, we explored the utility of the AIE dots of TTF for realtime two-photon intravital vasculature imaging. We compared oneand two-photon fluorescence of the AIE dots with different TTF loading ratios and studied their chemo- and photostabilities. The AIE dots were employed as contrast agents for in vivo two-photon fluorescence imaging for the first time. Unlike other nanomaterials such as graphene oxide (GO) nanoparticles ${ }^{7}$, the biocompatible AIE dots emit bright red light and can be intravenously injected into a mouse body from its tail vein to image the blood vessels at a deeper level of the brain. This enabled 3D micron-level resolution imaging as well as recording of the flow of the AIE dots in the blood vessels of the mouse brain with two-photon microscopy.

\section{Results}

Synthesis and characterization of AIE-dots. Fig. 2a shows the major steps of AIE-dots synthesis. During the mixing of solid lipidic mass and the added water under continuous sonication, the hydrophobic lipid segments of DSPE-mPEG ${ }_{5000}$ tended to be embedded in the hydrophobic TTF core and the hydrophilic PEG chains of DSPE$\mathrm{mPEG}_{5000}$ extended into the aqueous phase to render the nanodots with water dispersibility and excellent biocompatibility. In our experiments, different samples of AIE-dots aqueous suspension were prepared by mixing different amounts of DSPE-mPEG5000 with the constant amount of TTF $(0.25 \mathrm{mg})$ and constant volume of water $(2 \mathrm{~mL})$ added for micellization. The ratio of the weight of TTF to that of TTF and DSPE-mPEG5000 in the feed mixture was defined as TTF feeding ratio. For the preparation of TTF feeding ratio of $5 \mathrm{wt} \%, 10 \mathrm{wt} \%, 20 \mathrm{wt} \%, 30 \mathrm{wt} \%, 40 \mathrm{wt} \%, 50 \mathrm{wt} \%$, and $60 \mathrm{wt} \%$, the volume of DSPE-mPEG5000 solutions added was $4.75 \mathrm{~mL}$, $2.25 \mathrm{~mL}, 1 \mathrm{~mL}, 0.58 \mathrm{~mL}, 0.375 \mathrm{~mL}, 0.25 \mathrm{~mL}$ and $0.17 \mathrm{~mL}$, respectively. The amount of TTF molecules in the synthesized AIE-dots aqueous suspension was determined by their optical absorbance. The weight of the AIE-dots was calculated by the weight of TTF plus the weight of DSPE-mPEG5000 molecules added. The loading ratio of TTF in the AIE-dots was calculated by dividing the weight of the TTF in the aqueous suspension by the weight of the AIE-dots. The TTF encapsulation efficiency was calculated by dividing the TTF loading ratio by the TTF feeding ratio. Fig. $2 \mathrm{~b}$ shows the
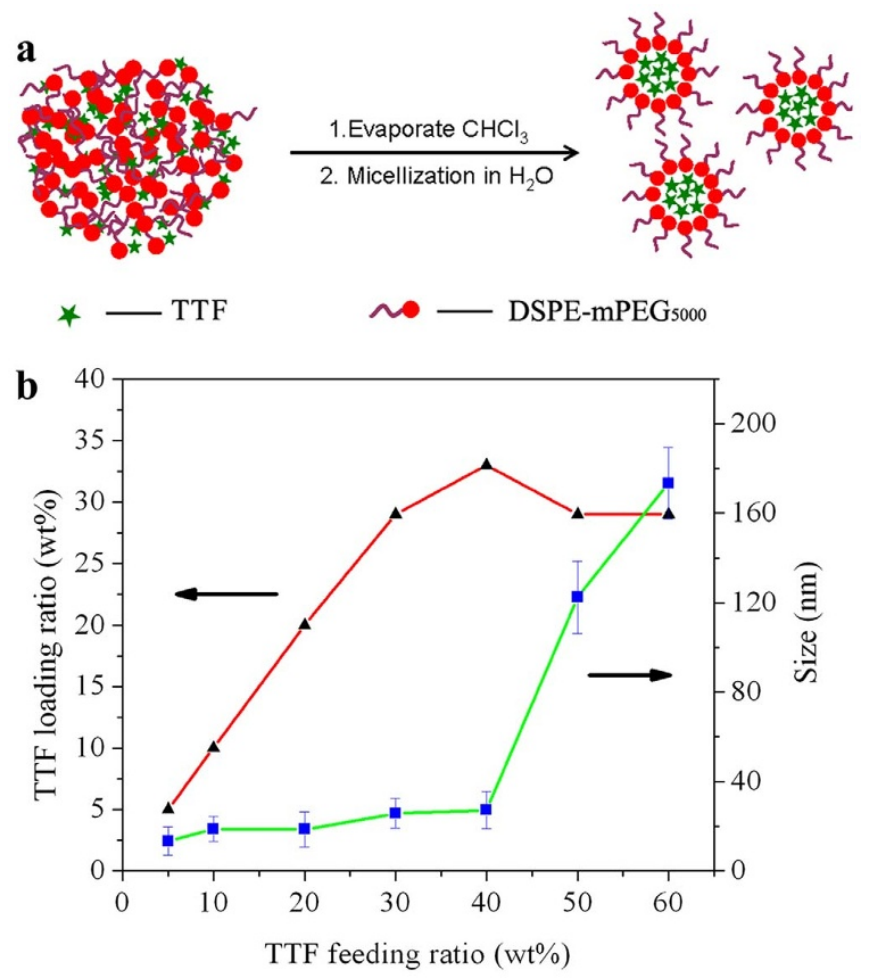

Figure $2 \mid$ Synthesis and characterization of AIE-dots. (a) A schematic illustration for the preparation of AIE-dots. (b) The loading ratio of AIEdots [the ratio of the total weight of TTF to that of AIE-dots in the aqueous suspension] and number-averaged particle size distribution of AIE-dots prepared with different feeding ratios [the ratio of the weight of TTF to that of TTF and DSPE-mPEG ${ }_{5000}$ in the feed mixture]. 

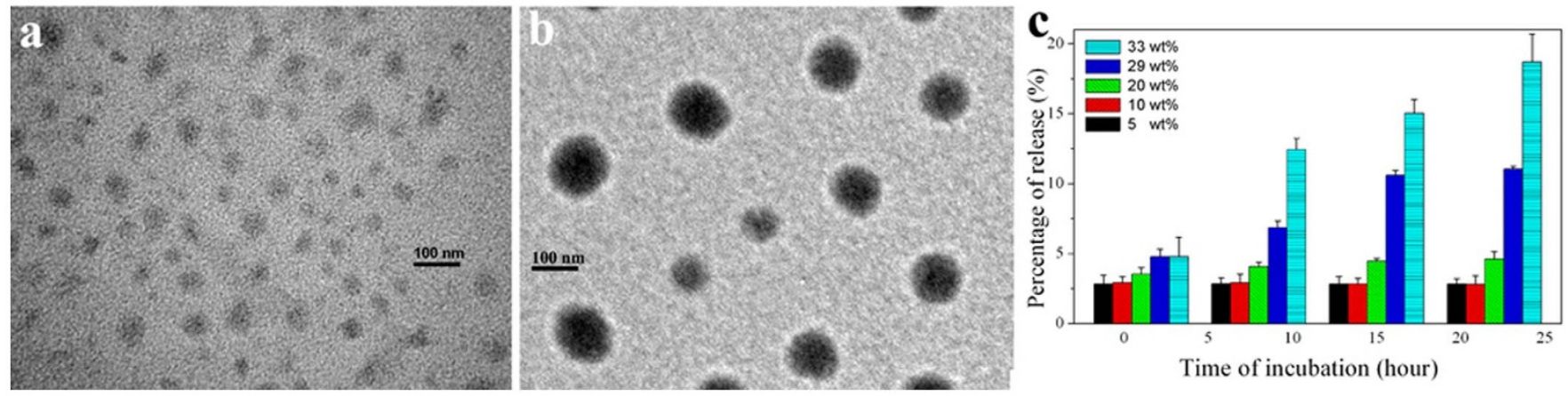

Figure 3 TEM and release kinetics studies of AIE-dots. (a and b) Representative TEM images of the AIE-dots prepared with 30 wt $\%$ (a) and $50 \mathrm{wt} \%$ (b) feeding ratio of TTF. (c) Release kinetics studies of AIE-dots with different loading ratio.

corresponding TTF loading ratio of AIE-dots prepared at different conditions of TTF feeding ratio. Our experimental results indicate an increased TTF loading ratio in the AIE-dots with an increase in the TTF feeding. The encapsulation efficiency of TTF was $>95 \mathrm{wt} \%$ when the TTF feeding ratio was $<30 \mathrm{wt} \%$. When the TTF feeding ratio increased to $>40 \mathrm{wt} \%$, the loading ratio of AIE-dots could not increase since the DSPE-mPEG5000 molecules was too few to encapsulate all the TTF molecules. Thus we observed a sudden decrease in the TTF encapsulation efficiency. The average diameters of AIE-dots were measured by using dynamic light scattering (DLS) technology. It is well known that particle size will increase if the surfactant concentration is decreased in the formulation of micellization progress. In our experiments, DSPEmPEG5000 molecules play the role of surfactant. Thus, in the situation of the TTF feeding ratio $<40 \mathrm{wt} \%$, we observed that particle size increased with decreasing of the amount of DSPEmPEG5000. However, when the TTF feeding ratio was $>40 \mathrm{wt} \%$, there was sudden increase in the average diameter of AIE-dots. We attributed the big difference in the average diameter of AIE-dots to the particle coagulation in the solution.

Typical TEM images of the AIE-dots with $30 \mathrm{wt} \%$ and $50 \mathrm{wt} \%$ feeding of TTF were shown in Fig. 3a and Fig. 3b. In our experiments, the loading ratio of these two kinds of AIE-dots was calculated to be the same of $29 \mathrm{wt} \%$. However, the TEM images indicated that the AIE-dots with $30 \mathrm{wt} \%$ feeding of TTF had an average size of less than $30 \mathrm{~nm}$ and the AIE-dots with $50 \mathrm{wt} \%$ feeding of TTF was larger than $100 \mathrm{~nm}$. In the cases of AIE-dots prepared with low TTF feeding ratios, the TTF loading ratio we obtained by dividing the weight of the total TTF by the total weight of AIE-dots in the aqueous suspension was approximately equal to the average component ratio of TTF in one AIE-dot. However, for the AIE-dots prepared with high TTF feeding ratios, particle coagulation occurred in the solution, and the loading ratio we obtained could not represent the average component ratio of TTF in one AIE-dot. Thus, even these two kinds of AIEdots are quite different in size, we obtained the same loading ratio result of $29 \mathrm{wt} \%$.

To study the stabilities of AIE-dots with different loading ratio, we tested possible leakage of the entrapped TTF molecules from the AIE dots. The results for the release kinetics studies of AIE-dots with different loading ratio are shown in Fig. 3c. When the AIE dots with low TTF contents (5-20 wt\%) were incubated with Tween-20 surfactant (1\%) at $37^{\circ} \mathrm{C}$ for $24 \mathrm{~h}$, the releases of TTF molecules from the AIE dots were insignificant $(<5 \mathrm{wt} \%)$. However, the release of TTF from the AIE dots with high TTF contents was significant.

Aggregation-enhanced fluorescence properties of AIE-dots. Fig. 4 shows the absorption and one-photon luminescence spectra of bare TTF molecules dissolved in $\mathrm{CHCl}_{3}$ and of AIE-dots with various TTF loading ratios. The absorption spectrum of the AIE dots suspended in water was similar to that of the TTF solution in chloroform, with an absorption peak at $500 \mathrm{~nm}$. The TTF solution showed a weak red fluorescence with an emission peak at $624 \mathrm{~nm}$. Its nanodots, however, emitted very strongly due to the AIE characteristics of the encapsulated TTF molecules. For aqueous suspensions of AIEdots, the TTF molecular concentration can be calculated as the total number of TTF molecular in the solution divided by the amount of the solution, and the AIE-dots concentration can be calculated as the total number of AIE-dot divided by the amount of the solution. In our experiment, we prepared aqueous suspension samples for AIEdots with loading ratio of $5 \mathrm{wt} \%, 10 \mathrm{wt} \%, 20 \mathrm{wt} \%, 29 \mathrm{wt} \%$ and $33 \mathrm{wt} \%$, respectively. For each sample, the absorbance at wavelength of $500 \mathrm{~nm}$ was kept the same as 0.08 , by adjusting the amount of AIE-dots added to the sample. Thus, the TTF concentration of each sample could be considered as the same, according to Beer's Law. We then measured the PL spectra of each sample, using a HITACHI F-2500 fluorescence spectrophotometer (excitation wavelength: $500 \mathrm{~nm}$ ). Since the concentrations of TTF molecules were the same in all samples, the PL spectra could be considered as obtained from the same amount of TTF molecules. As shown in Fig. 4, we detected higher PL intensities from the samples with higher TTF lading ratios. We attributed these results to the aggregation enhanced fluorescence of TTF molecules.

In our previous studies, it has been proved that the AIE dots of TTF possess large two-photon absorption cross sections, which can result in high signal-to-noise ratios in bioimaging measurements,

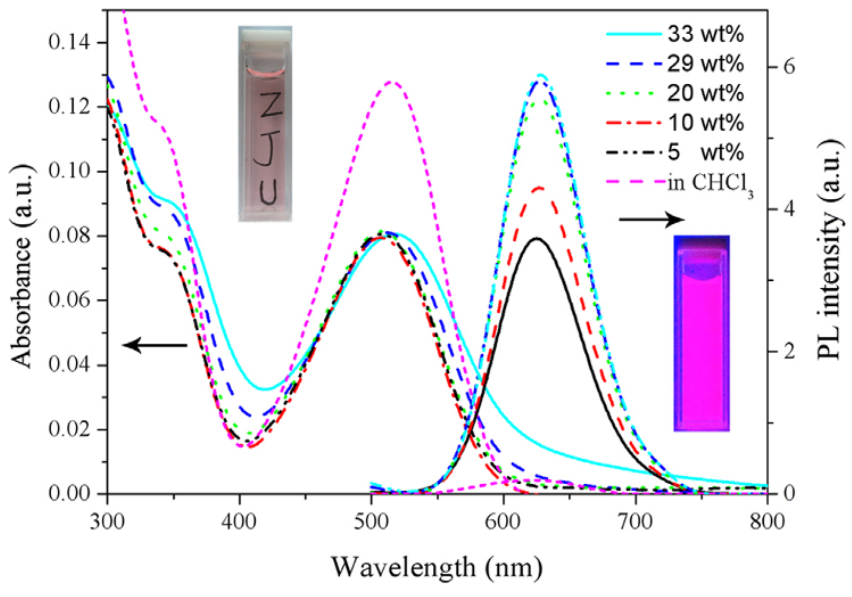

Figure $4 \mid$ Quantitative comparison of the macroscopic photoluminescence (PL) from AIE-dots with various TTF loading ratios. Absorbance and one-photon luminescence spectra of bare TTF molecules dissolved in $\mathrm{CHCl}_{3}$ and of AIE-dots with various TTF loading ratios ( $5 \mathrm{wt} \%, 10 \mathrm{wt} \%, 20 \mathrm{wt} \%, 29 \mathrm{wt} \%, 33 \mathrm{wt} \%)$ suspended in water. Inset: Pictures of AIE-dots aqueous suspension under visible light (left) and ultraviolet light excitation (right) (365 nm). 
and the brightness of their two-photon fluorescence is comparable or higher than that of QDs or other organic nanoaggregates in water ${ }^{22}$. In the present work, we quantitatively compared the signals of the microcosmic one- (1 PL) and two-photon luminescence (2 PL) from the AIE dots with different TTF contents. The aqueous suspensions of the AIE dots were dropped onto glass slides and imaged with a laser scanning confocal microscope. A $543 \mathrm{~nm}$ CW laser and an $800 \mathrm{~nm}$ fs laser were used as excitation sources to perform one(1 PL) and two-photon luminescence (2 PL), respectively. A water-immersion objective lens $(20 \times, \mathrm{NA}=0.75)$ and a filter ranging from $570 \mathrm{~nm}$ to $670 \mathrm{~nm}$ were adopted to extract the fluorescence signals from AIE-dots. The specifics of the microscope for one and two-photon fluorescence imaging were kept the same, respectively. The specifics of the microscope for $1 \mathrm{PL}$ and $2 \mathrm{PL}$ imaging were kept the same, respectively. Fig. 5 shows the microcosmic $1 \mathrm{PL}$ and 2 PL from AIE dots with various TTF loading ratios. As the suspensions were highly dilute, the fluorescence signal from a single spot could be regarded as the emission from a single AIE dot. However, the fluorescence intensity fluctuated in each image. We attributed the fluctuated fluorescence intensity to the random dispersion of AIE-dots in solutions, and the fluorescence signals were generated by AIE-dots at different planes of each sample. Through statistics and analysis of the fluorescence images, we obtained an average intensity for each picture which can be used to reflect the fluorescence intensity for single dot. The intensity distributions and average intensity of each picture are given under each image shown in Fig. 5. These results demonstrated that AIE dots with a higher TTF content emit more intensely (for both $1 \mathrm{PL}$ and $2 \mathrm{PL}$ ) than those with a lower TTF content.
Considering the emission brightness and chemical stability, the AIE dots with $20 \mathrm{wt} \%$ of TTF is an optimal choice for bioimaging applications. Since the photostability is of importance for bioimaging, we tested the 2 PL strength changes of AIE-dots irradiated by a femtosecond laser with an extended period of time. From the results shown in Fig. 6, for AIE-dots with 20 wt\% of TTF, the 2 PL strength did not change much after the AIE dots had been irradiated with a femtosecond laser for 10 minutes. The high photostability is an added bonus that enables the TTF-encapsulated AIE dots to work as an excellent optical bioprobe for in vivo two-photon microscopic fluorescence imaging.

Biodistribution of AIE-dots in mice. To verify the distribution of AIE-dots in mice, we injected them intravenously (tail vein) in male $\mathrm{BALB} / \mathrm{c}$ mice. Major organs including heart, liver, spleen, lungs and kidney were resected after different times and imaged immediately using a Maestro optical imaging system (CRI, Inc. Woburn, MA). From the results shown in Fig. 7, we can see that the fluorescence of AIE-dots could be observed in all the organs $3 \mathrm{~h}$ and $6 \mathrm{~h}$ postinjection, indicating that AIE-dots were flowing in the circulatory system of mice. It is quite evident that as time went by, the major accumulation of these AIE-dots occurs predominantly in liver (Fig. 7c and Fig. 7d), which was attributed to the metabolic functions of animals.

AIE-dots for imaging of ear blood vessels. The good 2 PL performance of the AIE dots prompted us to apply them to blood vessel imaging of live mice employing the TPLSM technique. The mice were intravenously injected with the suspensions of the AIE dots
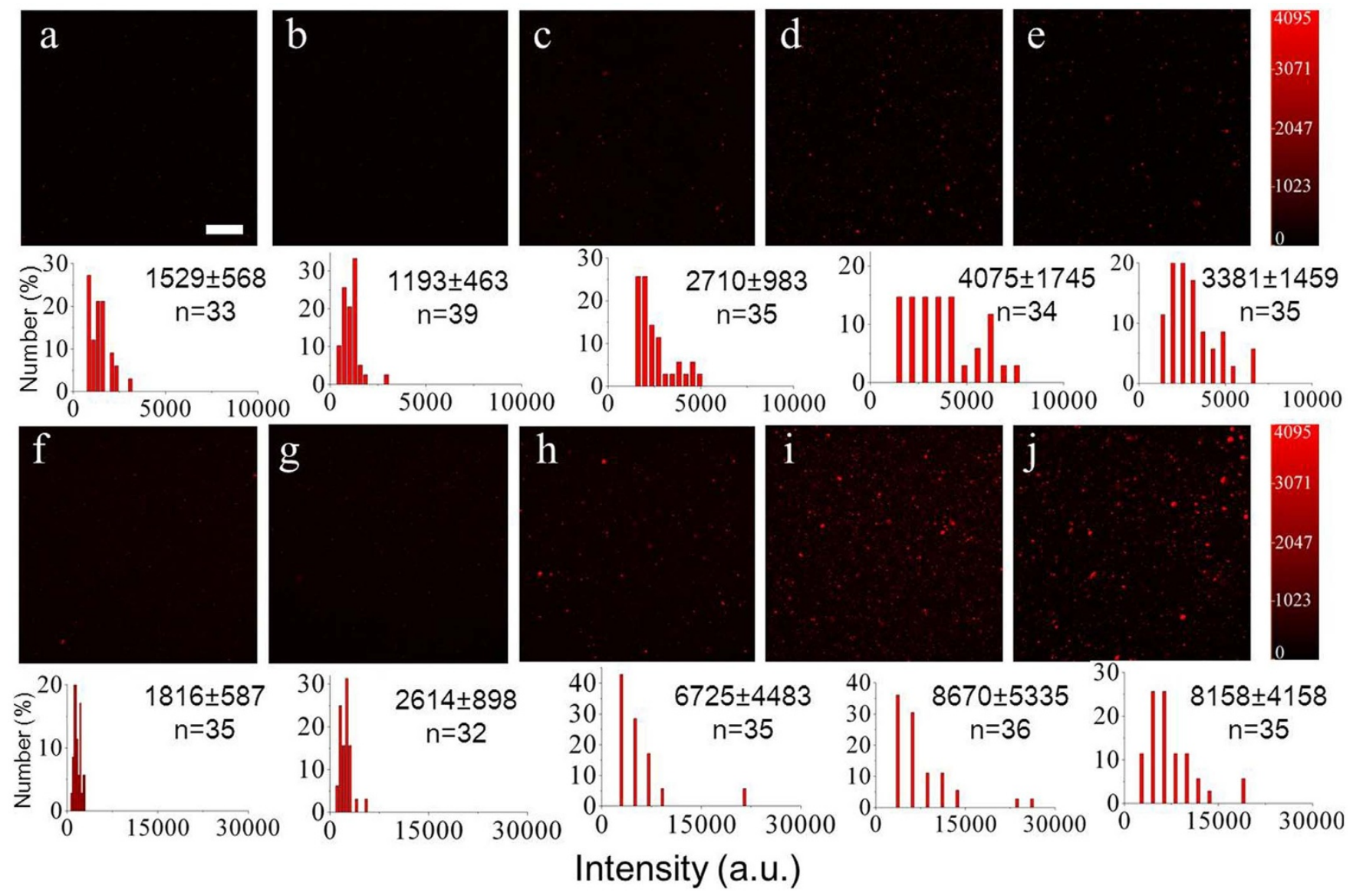

Figure 5 Quantitative comparison of the microcosmic one- (1 PL) and two-photon luminescence (2 PL) from AIE dots with various TTF loading

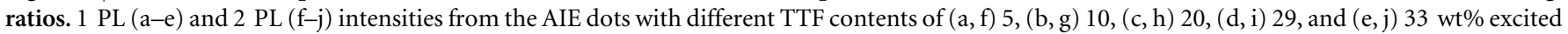
by a CW laser at $543 \mathrm{~nm}$ and a femtosecond laser at $800 \mathrm{~nm}$, respectively. Scale bars: $100 \mu \mathrm{m}$. Intensity distribution is shown under each image. 

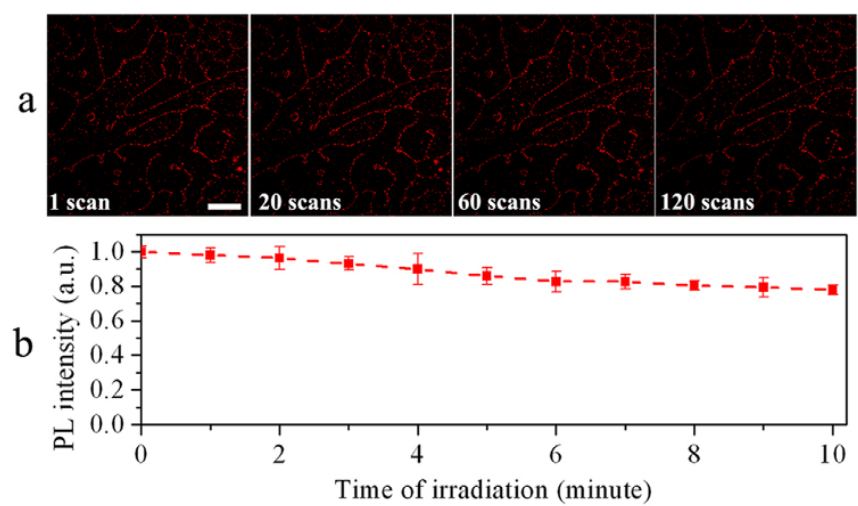

Figure 6 | Studies of two-photon excited photostability of AIE-dots.

(a) 2 PL images of AIE-dots after being scanned for various times. An objective lens of $20 \times$ and NA $=0.75$ was used. Scale bar: $100 \mu \mathrm{m}$. (b) PL intensities of the samples at different time points of irradiation.

(20 wt $\%$ TTF content, $1 \mathrm{mg} / \mathrm{mL}$ in $1 \times \mathrm{PBS}, 100 \mu \mathrm{L}$ solution per mouse). Mice were immediately placed on a Petri dish with one ear attached to the coverslip. An upright Olympus laser scanning confocal microscope (FV1000) was used for both one-photon and two-photon luminescence imaging experiments. The instrument and instrument set up of fluorescence signal collecting for both $1 \mathrm{~L}$ and 2 PL imaging are the same. After injected AIE-dots for $30 \mathrm{~min}$, bright 2 PL signals (Fig. 8c) were observed in the ear blood vessels of the mice upon femtosecond laser excitation (800 nm, $100 \mathrm{fs}$ ), while no 1 PL signals (Fig. 7b) from the AIE dots in the vessels could be detected when excited by a CW laser at $543 \mathrm{~nm}$. It was mainly attributed to the absorption/scattering loss of $543 \mathrm{~nm}$ excitation in skin/blood, which greatly limited the imaging depth of in vivo onephoton confocal microscopy. The merged images (Fig. 8d) of bright field image (Fig. 8a) and fluorescence images (Fig. 8b and Fig. 8c) confirmed the 2 PL signals of AIE-dots flowing in the ear blood vessels. For the control experiments of the blood vessel imaging, mice injected with DSPE-PEG solution $(1 \mathrm{mg} / \mathrm{mL}$ in $1 \times \mathrm{PBS}$, $100 \mu \mathrm{L}$ solutions per mouse) were studied and neither $1 \mathrm{PL}$ nor 2 PL signals could be detected (data not shown), showing that the $2 \mathrm{PL}$ in the experiment was emitted by the AIE-dots rather than the tissues or blood in the mice's ears. According a method reported by Chaigneau et al ${ }^{24}$, intravenous injection of fluorescein dextran could revealed the vascular architecture as well as individual RBCs by using a rapid line scan along the capillary axis, which is one of the functions of the commercial confocal microscope software. In our experiments, the injected AIE-dots flowed in the blood plasma and could not get into the red blood cells due to the existing of red cellmembranes. Thus, individual RBCs appeared as shadows flowing with the fluorescent plasma during the fluorescence imaging. Fig. 8e shows the instantaneous velocity $(\mathrm{dx} / \mathrm{dT})$ of each $\mathrm{RBC}$ by using AIE-dots as fluorescent probes in plasma. To study the blood circulation kinetics of AIE-dots in mice, we measured the 2 PL intensity from the same region of the blood vessel at different time points after injection for each mouse. The concentration of AIE-dots in blood was represented by their 2 PL intensity and was normalized by the first time point. Each data point shown in Fig. $8 \mathrm{f}$ was an average from 5 mice. A blood circulation half-life of $\sim 4 \mathrm{~h}$ was observed for AIE-dots by our experimental results shown in Fig. 8f.

AIE-dots for imaging of brain blood vessels. The deep tissue imaging capability, high spatial resolution, and low thermal damage to biological samples make TPLSM very useful for brain imaging of small animals. In the past few years, two-photon brain imaging has been widely applied in various areas of biological research, such as neurobehavioral analysis/regulation, gene therapy in the brain, and blood brain barrier penetration ${ }^{31,32}$. Usually, the fluorophores used
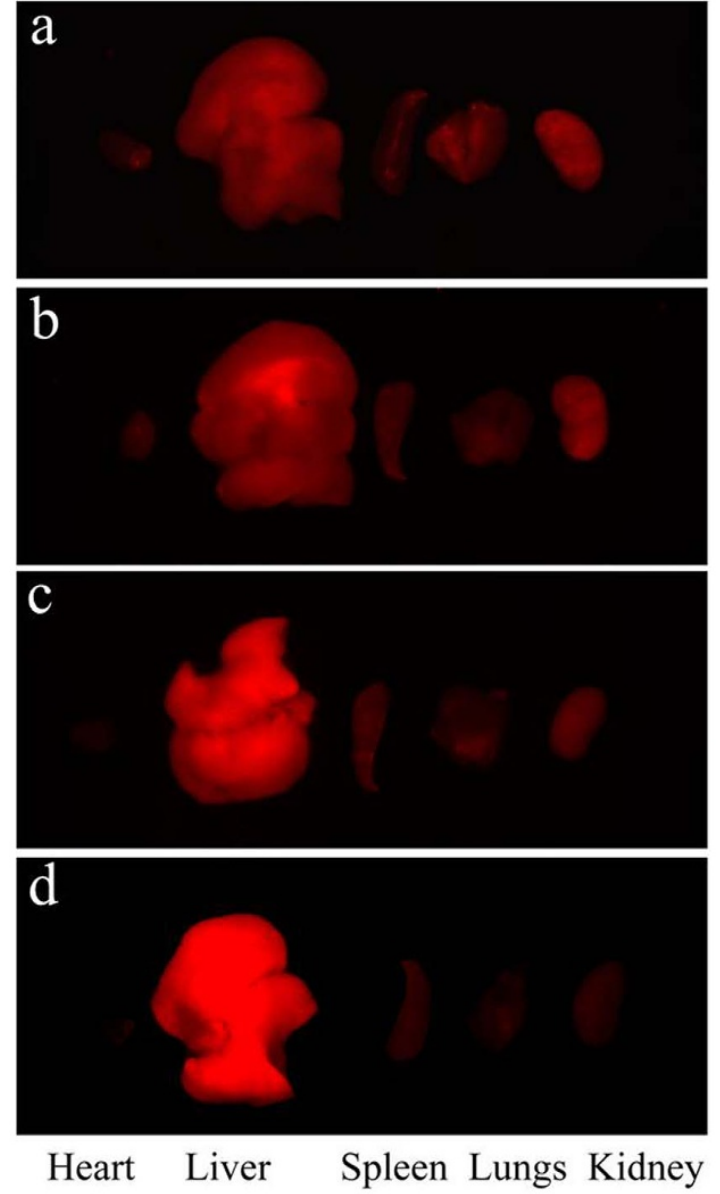

Figure 7 Biodistribution studies of AIE-dots in mice. Ex vivo fluorescence images of the major organs (heart, liver, spleen, lungs and kidney) resected from mice (a) $3 \mathrm{~h}$, (b) $6 \mathrm{~h}$, (c) $16 \mathrm{~h}$, (d) $24 \mathrm{~h}$ after injection of AIE-dots (with $20 \mathrm{wt} \%$ TTF loading, $1 \mathrm{mg} / \mathrm{mL}$ in $1 \times \mathrm{PBS}$, $100 \mu \mathrm{L}$ solutions per mouse).

for the brain imaging are gene-transfected or commercial products. The former is cumbersome and time-consuming in process, while the latter is limited by the notorious ACQ effect when its concentration becomes high. Recently, we reported the use of graphene oxide (GO) nanoparticles as fluorescent probes for twophoton brain imaging through microinjection of GO into the mouse brain $^{7}$. In some literatures, GO was considered as biocompatible fluorescent probes $^{33-35}$. However, the emission of the GO nanoparticles, is in the green spectral region and their fluorescence quantum yields are not as high as those of the AIE dots. The red fluorescent AIE dots are thus more suitable for in vivo staining and deep imaging of brain blood vessels. The AIE dots only need to be injected intravenously into animal body for blood circulation, which is facile and can be easily controlled. A 3D mixed image of the AIE dots in the blood vessels of the brain of a mouse was reconstructed from the TPLSM data (Fig. 9a). The imaged region $(508 \mu \mathrm{m} \times$ $508 \mu \mathrm{m} \times 125 \mu \mathrm{m})$ was at a depth range of $175-300 \mu \mathrm{m}$ in the mouse brain (Fig. 9b-e). Since the 2 PL of the AIE dots is very distinct, the intravenously injected AIE dots can reveal the vascular architecture in the mouse brain. The performances (e.g., flow and penetration) of the AIE dots in the blood vessel can be readily manifested by the 2 PL signals. The video obtained from the twophoton fluorescence images dynamically and vividly illustrate the flow process of the AIE dots in the blood vessel of the brain (see the Supporting Information). 

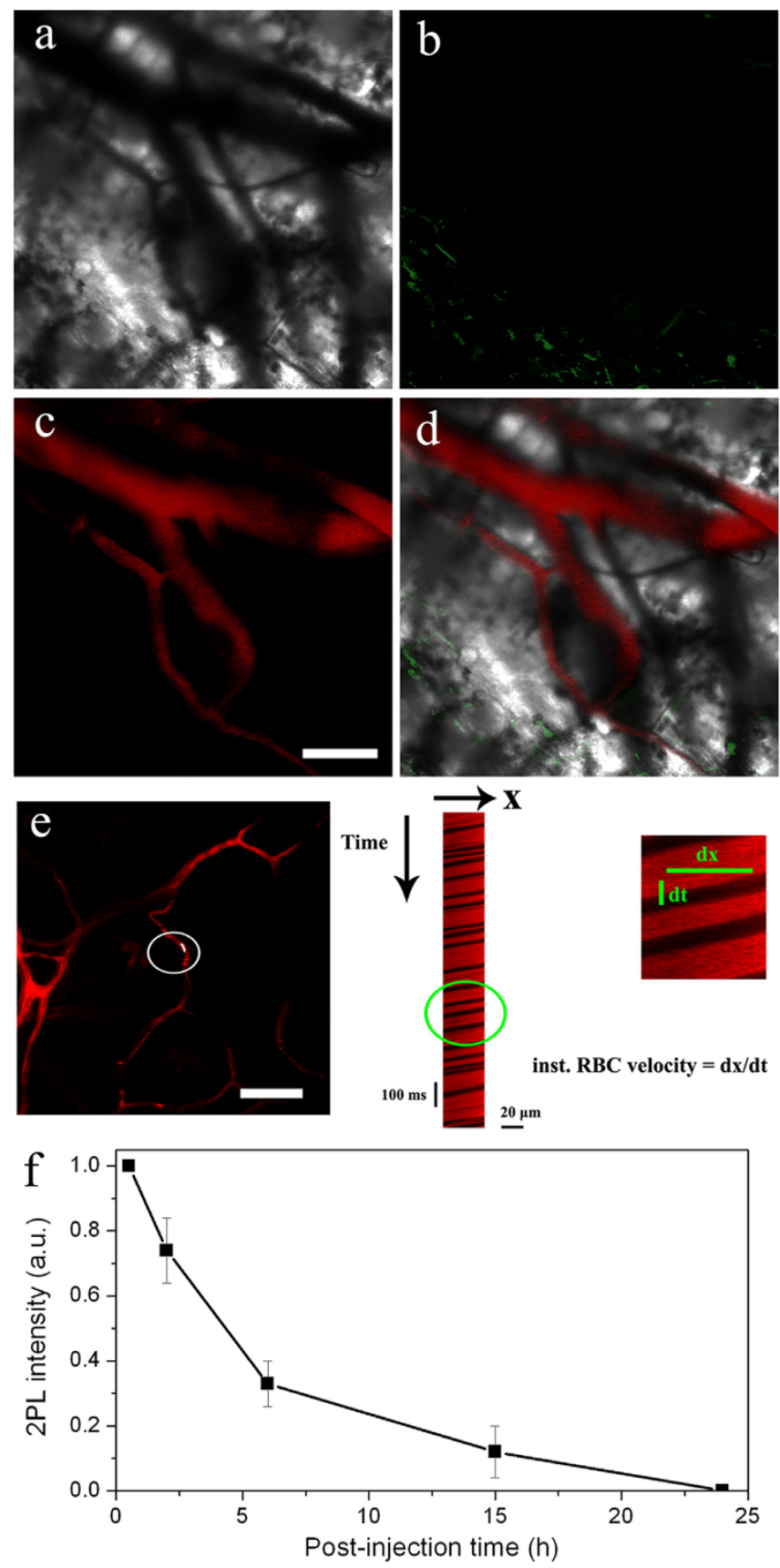

Figure 8 Imaging of ear blood vessels of a mouse using AIE-dots. (a-d) Bright field (a), one-photon confocal luminescence (b) two-photon scanning luminescence (c), and merged images of the intravenously injected AIE dots in the blood vessel of the ear of a mouse (d). These images were taken 30 minutes after the dots injection. (e) A line scan along capillaries of a mouse brain was used to observe RBC flow and determine its instantaneous velocity $(\mathrm{dx} / \mathrm{dT})$ with two-photon confocal microscope. Scale bar: $100 \mu \mathrm{m}$. (f) Blood circulation kinetics of AIE-dots in mice.

\section{Discussions}

Very recently, Ding et al performed two-photon imaging of ear and brain blood vessels, using a kind of AIE-dots with green emission ${ }^{36}$. Compared with the AIE nanoparticle reported in that work, the TTFencapsulated AIE dots are more benefit for bioimaging due to their longer wavelength emission. In this paper, we have demonstrated $1 \mathrm{PL}$ and 2 PL of the TTF-encapsulated AIE dots and proven their photostable feature for the 2 PL bioimaging. The TPLSM imaging enabled reconstruction of $3 \mathrm{D}$ distribution of the AIE dots intravenously injected into the blood vessels of a mouse brain. The flow of the red AIE dots could also be dynamically monitored in vivo. We also studied the biodistribution and the blood circulation kinetics of the AIE-dots in mice, which are important and helpful for further applications of AIE-dots in animals. Further efforts will be made to optimize femtosecond laser excitation and optical imaging systems to achieve even clearer and deeper bioimaging and to realize the full potential of the AIE dots for in vivo bioimaging applications in the areas of neurobehavioral analysis/regulation, gene therapy in the brain, barrier penetration through brain blood capillaries, etc.

\section{Methods}

Materials and instruments. The TTF fluorogen was prepared following our previously published protocol ${ }^{22}$. DSPE- $\mathrm{mPEG}_{5000}$ was purchased from Creative PEGWorks, Inc. Other chemical reagents, which were not specially pointed out, were obtained from Sigma Inc. Deioned (DI) water was used in all the experimental procedures. A Shimadzu 2550 UV-vis scanning spectrophotometer and a HITACHI F-2500 fluorescence spectrophotometer were used to measure the absorption and photoluminescence (PL) spectra of samples. Dynamic light scattering (DLS) analysis was conducted using a Dawn ${ }^{\circledR}$ Heleos light scatter from Wyatt Technology Corp at room temperature. TEM images were taken by a JEOL JEM-1230 transmission electron microscope operating at $160 \mathrm{kV}$ in bright-field mode.

Preparation and characterization of AIE-dots. AIE dots were fabricated through a modified nanoprecipitation procedure ${ }^{10}$. Briefly, $0.25 \mathrm{~mL}$ of TTF solutions in chloroform $(1 \mathrm{mg} / \mathrm{mL})$ and a certain amount of DSPE-mPEG 5000 solutions in chloroform $(1 \mathrm{mg} / \mathrm{mL})$ were mixed in a flask $(25 \mathrm{~mL})$. The mixture was sonicated for several minutes to form a homogeneous red solution and dried under vacuum in a rotary evaporator at $70^{\circ} \mathrm{C}$. After the chloroform was completely removed, $2 \mathrm{~mL}$ of deionized water was added into the flask and the solution was sonicated for $2 \mathrm{~min}$. Subsequently, an optically clear suspension containing AIE-dots was prepared. By varying the amount of DSPE-mPEG ${ }_{5000}$ added into the mixture, AIE-dots with different TTF feeding ratios were prepared. For the preparation of TTF feeding ratio of $5 \mathrm{wt} \%, 10 \mathrm{wt} \%, 20 \mathrm{wt} \%, 30 \mathrm{wt} \%, 40 \mathrm{wt} \%, 50$, and $60 \mathrm{wt} \%$, the volume of DSPE$\mathrm{mPEG}_{5000}$ solutions added was $4.75 \mathrm{~mL}, 2.25 \mathrm{~mL}, 1 \mathrm{~mL}, 0.58 \mathrm{~mL}, 0.375 \mathrm{~mL}$, $0.25 \mathrm{~mL}$ and $0.17 \mathrm{~mL}$, respectively.

To determine the loading ratio of TTF in AIE-dots, solutions of AIE-dots were measured by a Shimadzu 2550 UV-vis scanning spectrophotometer. The TTF concentration was determined by comparing its absorption intensity with that of a sample with known concentration at $500 \mathrm{~nm}$. The feeding ratio was calculated by dividing the weight of TTF by the total weight of TTF and DSPE-mPEG ${ }_{5000}$ in the feed mixture. The loading ratio was calculated by dividing the weight of the loaded TTF by the weight of the AIE-dots. The encapsulation efficiency was calculated by dividing the loading ratio by the feeding ratio. The average sizes of AIE-dots were measured by Dynamic Light Scattering (DLS) analysis.

For release kinetics studies, solutions of AIE-dots were incubated with $1 \mathrm{~mL}$ Tween-20 solutions ( $1 \%$ in DI water) at $40^{\circ} \mathrm{C}$. After a certain time, the sample was spin-filtered using microfuge membrane-filter (NANOSEP $100 \mathrm{~K}$ OMEGA, Pall Corporation, USA) at 12,000 rpm for $15 \mathrm{~min}$ (spin filtration). The filtrated solution, which passed through the membrane, was collected. Its extinction spectrum, as well as that of the original aqueous solution of AIE-dots, was measured. The ratio of the two extinction peak intensities could be used to characterize the release percentage of TTF from AIE-dots.

One- and two-photon fluorescence of AIE-dots. To compare the microcosmic onephoton luminescence (1 PL) and two-photon luminescence (2 PL) signals quantitatively from AIE-dots with different TTF loading ratios, aqueous suspensions of AIE-dots with different TTF loading ratios were dropped on glass slides and imaged with a laser scanning confocal microscope (Olympus, BX61W1-FV1000). The $1 \mathrm{PL}$ and $2 \mathrm{PL}$ of samples were performed by a CW laser at $543 \mathrm{~nm}$ and a femtosecond laser at $800 \mathrm{~nm}$, respectively. A water-immersion objective lens $(20 \times$, $\mathrm{NA}=0.75$ ) was used to focus the laser beam onto the samples, and an external detector was used to collect emission signals. Through statistics and analysis of a mass of spots from the fluorescence images, we obtained an average intensity for each picture which can be used to reflect the fluorescence intensity for single dot.

Animals. Male black mice (C57 line) were obtained from the Laboratory Animal Center of Zhejiang University (Hangzhou, China). Mice were housed in cages in groups ( 5 mice per cage) and fed with standard mouse chow and water. The cages were maintained in a room with controlled temperature $\left(25 \pm 1^{\circ} \mathrm{C}\right)$ and a $12 \mathrm{~h}$ light/ dark cycle. The protocol of animal experiments was approved by the Institutional Ethical Committee of Animal Experimentation of Zhejiang University in China, and the experiments were performed strictly according to governmental and international guidelines on animal experimentation. According to requirements for Biosafety and Animal Ethics, all efforts were made to minimize the number of animals used and their suffering.

Biodistribution of AIE-dots in mice. Mice were intravenously injected with suspensions of AIE-dots (with $20 \mathrm{wt} \%$ TTF loading, $1 \mathrm{mg} / \mathrm{mL}$ in $1 \times$ phosphate buffered saline (PBS), $100 \mu \mathrm{L}$ solutions per mouse). The mice were put down various 

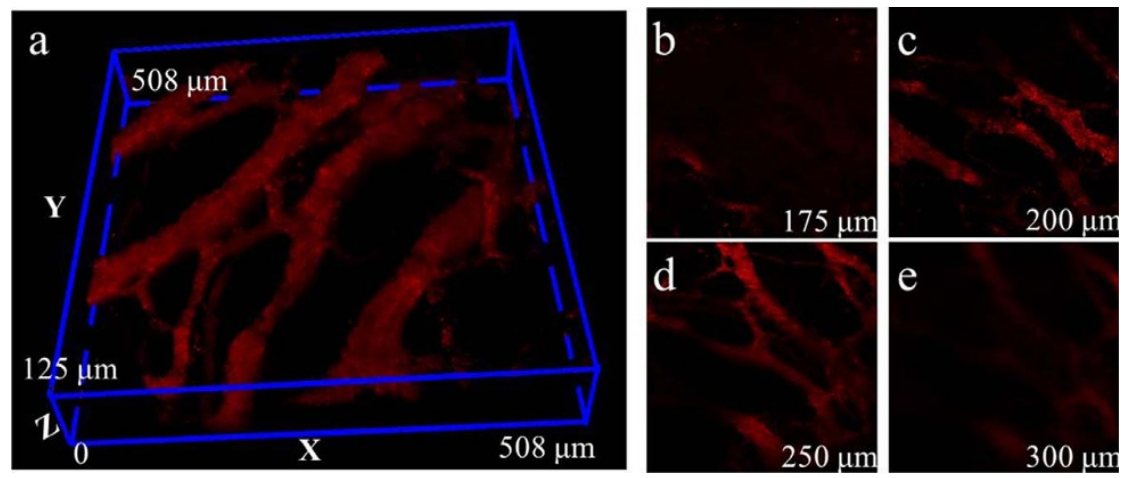

Figure 9 Imaging of brain blood vessels of a mouse using AIE-dots. (a) A reconstructed 3D image showing the distribution of the AIE-dots in the blood vessels at a depth range of 175-300 $\mu \mathrm{m}$ of the brain of a mouse. (b-e) Individual images taken at the depths of $175 \mu \mathrm{m}(\mathrm{b}), 200 \mu \mathrm{m}(\mathrm{c}), 250 \mu \mathrm{m}(\mathrm{d})$, and $300 \mu \mathrm{m}(\mathrm{e})$. These data were recorded 30 minutes post-injection by a two-photon scanning microscope.

hours after injection. Three mice were used at each time point after injection. Their major organs were taken out and imaged by utilizing a Maestro in vivo optical imaging system (CRI, Inc. Woburn, MA). The organs of mice were excited with laser of $595 \mathrm{~nm}$ peak wavelength. The fluorescence images were captured with a constant exposure time of $2000 \mathrm{~ms}$ for all the samples.

In vivo imaging of ear blood vessels. Six mice were randomly divided into two groups. Three mice in one group were intravenously injected with suspensions of AIE-dots (with 20 wt\% TTF loading, $1 \mathrm{mg} / \mathrm{mL}$ in $1 \times$ PBS, $100 \mu \mathrm{L}$ solutions per mouse) as experimental group, and the mice in the other group was intravenously injected with PBS solution (100 $\mu \mathrm{L}$ solutions per mouse) as control group. For ear blood vessels imaging, the mice were anaesthetized with an intraperitoneal injection of $0.5 \%$ pentobarbital sodium and placed on a Petri dish with one ear attached to the coverslip. An upright two-photon scanning microscope (Olympus, BX61W1FV1000) was used for 2 PL imaging. A long work distance (objective length $=2 \mathrm{~mm}$ ) water-immersion objective lens $(25 \times, \mathrm{NA}=1.05)$ was used to focus the laser beam onto the water-immersed earlobe, and an external photomultiplier tube (PMT) was used to collect two-photon induced emission signals via non-descanned detection (NDD).

In vivo imaging of brain blood vessels. Six mice were random divided into two groups. Three mice in one group were intravenously injected with suspensions of AIE-dots (with $20 \mathrm{wt} \%$ TTF loading, $1 \mathrm{mg} / \mathrm{mL}$ in $1 \times \mathrm{PBS}, 100 \mu \mathrm{L}$ solutions per mouse) as experimental group, and the mice in the other group was intravenously injected with PBS solution ( $100 \mu \mathrm{L}$ solutions per mouse) as control group. For in vivo brain imaging of mice, the mice were anaesthetized with an intraperitoneal injection of $0.5 \%$ pentobarbital sodium and their skulls were opened up through microsurgery. An upright two-photon scanning microscope was used to image the blood vessels of the mice. For the description of the immobilization of mice's heads and how the objective of the upright two-photon scanning microscope was arranged to contact the brain, we refer to our previous work [ref. 7].

1. Prasad, P. N. Introduction to Biophotonics (Wiley-Interscience, New York, 2004).

2. Kim, S., Pudavar, H. E., Bonoiu, A. \& Prasad, P. N. Aggregation-enhanced fluorescence in organically modified silica nanoparticles: A novel approach toward high-signal-output nanoprobes for two-photon fluorescence bioimaging. Adv. Mater. 19, 3791-3795 (2007).

3. Qian, J. et al. Photosensitizer encapsulated organically modified silica nanoparticles for direct two-photon photodynamic therapy and in vivo functional imaging. Biomaterials 33, 4851-4860 (2012).

4. Larson, D. R. et al. Water-soluble quantum dots for multiphoton fluorescence imaging in vivo. Science 300, 1434-1436 (2003).

5. Wang, H. F. et al. In vitro and in vivo two-photon luminescence imaging of single gold nanorods. P. Natl. Acad. Sci. USA 102, 15752-15756 (2005).

6. Tong, L., He, W., Zhang, Y., Zheng, W. \& Cheng, J. X. Visualizing systemic clearance and cellular level biodistribution of goldnanorods by intrinsic twophoton luminescence. Langmuir 25, 12454-12459 (2009).

7. Qian, J. Observation of multiphoton-induced fluorescence from grapheneoxide nanoparticles and applications in in vivo functional bioimaging. Angew. Chem. Int. Ed. 51, 10570-10575 (2012).

8. Li, J. et al. Graphene oxide nanoparticles as a nonbleaching optical probe for twophoton luminescence imaging and cell therapy. Angew. Chem. Int. Ed. 51, 1830-1834 (2012).

9. Mérian, J., Gravier, J., Navarro, F. \& Texier, I. Fluorescent nanoprobes dedicated to in vivo imaging: from preclinical validations to clinical translation. Molecules 17, 5564-5591 (2012).

10. Wang, D. et al. Aggregation-enhanced fluorescence in PEGylated phospholipid nanomicelles for in vivo imaging. Biomaterials 32, 5880-5888 (2011).
11. Jiang, S., Gnanasammandhan, M. K. \& Zhang, Y. Optical imaging-guided cancer therapy with fluorescent nanoparticles. J. R. Soc. Interface 7, 3-18 (2010).

12. Birks, J. B. Photophysics of Aromatic Molecules (Wiley, London, 1970).

13. Luo, J. et al. Aggregation-induced emission of 1-methyl-1,2,3,4,5pentaphenylsilole. Chem. Commun. 1740-1741 (2001).

14. Ding, D., Li, K., Liu, B. \& Tang, B. Z. Bioprobes Based on AIE Fluorogens. Acc. Chem. Res. 46, DOI: 10.1021/ar3003464 (2013).

15. Qin, A., Lam, J. W. Y. \& Tang, B. Z. Luminogenic polymers with aggregationinduced emission characteristics. Prog. Polym. Sci. 37, 182-209 (2012).

16. Kim, S. H., Huang, H., Pudavar, H. E., Cui, Y. P. \& Prasad, P. N. Intraparticle energy transfer and fluorescence photoconversion in nanoparticles: An optical highlighter nanoprobe for two-photon bioimaging. Chem. Mater. 19, 5650-5656 (2007).

17. Kim, S., Ohulchanskyy, T. Y., Pudavar, H. E., Pandey, R. K. \& Prasad, P. N. Organically modified silica nanoparticles co-encapsulating photosensitizing drug and aggregation-enhanced two-photon absorbing fluorescent dye aggregates for two-photon photodynamic therapy. J. Am. Chem. Soc. 129, 2669-2675 (2007).

18. Qin, W. et al. Biocompatible nanoparticles with aggregation-induced emission characteristics as far-red/near-infrared fluorescent bioprobes for in vitro and in vivoimaging applications. Adv. Funct. Mater. 22, 771-779 (2012).

19. Wu, W. C. et al. Enhancement of aggregation-induced emission in dyeencapsulating polymeric micelles for bioimaging. Adv. Func. Mater. 20, 1413-1423 (2010).

20. Wang, X. H. et al. Folatereceptor-targeted aggregation-enhanced near-IR emitting silica nanoprobe for one-photon in vivo and two-photon ex vivo fluorescence bioimaging. Bioconjug. Chem. 22, 1438-1450 (2010).

21. Yu, Y. et al. Cytophilicfluorescent bioprobes for long-term cell tracking. $A d v$. Mater. 23, 3298-3302 (2011)

22. Li, K. et al. Photostable fluorescent organic dots with aggregation-induced emission (AIE dots) for noninvasive long-term cell tracing. Sci. Rep. 3, 1150 DOI:10.1038/srep01150 (2013).

23. Geng, J. et al. Eccentric loading of fluorogen with aggregation-induced emission in PLGA matrix increases nanoparticle fluorescence quantum yield for targeted cellular imaging. Small 9, 2012-2019 (2013).

24. Chaigneau, E., Oheim, M., Audinat, E. \& Charpak, S. Two-photon imaging of capillary blood flow in olfactory bulb glomeruli. Proc. Natl. Acad. Sci. USA 100, 13081-13086 (2003).

25. Krishna, T. R. et al. Water-soluble dendrimeric two-photon tracers for in vivo imaging. Angew. Chem. Int. Ed. 45, 4645-4648 (2006).

26. Misgeld, T. \& Kerschensteiner, M. In vivo imaging of the diseased nervous system. Nat. Rev. Neurosci. 7, 449-463 (2006).

27. Weissleder, R., \& Ntziachristos, V. Shedding light onto live molecular targets. Nat. Med. 9, 123-128 (2003).

28. Dubertret, B. et al. In vivo imaging of quantum dots encapsulated in phospholipid micelles. Science 298, 1759-1762 (2002).

29. Ashok, B., Arleth, L., Hjelm, R. P., Rubinstein, I. \& Önyuksel, H. In vitro characterization of PEGylated phospholipid micelles for improved drug solubilization: Effects of PEG chain length and PC incorporation. J. Pharm. Sci. 93, 2476-2487 (2004).

30. Joralemon, M. J., McRae, S. \& Emrick, T. PEGylated polymers for medicine: from conjugation to self-assembled systems. Chem. Commun 46, 1377-1393 (2010).

31. Bonoiu, A. C. et al. Nanotechnology approach for drug addiction therapy: Gene silencing using delivery of gold nanorod-siRNA nanoplex in dopaminergic neurons. Proc. Natl. Acad. Sci. USA 106, 5546-5550 (2009).

32. Bharali, D. J. et al. Organically modified silica nanoparticles: A nonviral vector for in vivo gene delivery and expression in the brain. P. Natl. Acad. Sci. USA 102, 11539-11544 (2005)

33. Sun, X. et al. Nano-graphene oxide for cellular imaging and drug delivery. Nano Res. 1, 203-212 (2008). 
34. Yang, K. et al. Graphene in mice: ultrahigh in vivo tumor uptake and efficient photothermal therapy. Nano Lett. 10, 3318-3323 (2010).

35. Li, J. et al. "Graphene oxide nanoparticles as a nonbleaching optical probe for twophoton luminescence imaging and cell therapy." Angew. Chem. Int. Ed. 51, 1830-1834 (2012).

36. Ding, D. et al. Ultrabright organic dots with aggregation-induced emission characteristics for real-time two-photon intravital vasculature imaging. $A d v$. Mater. 25, 6083-6088 (2013).

\section{Acknowledgments}

The work reported in this paper was partially supported by the Ministry of Science and Technology of China (973 Program; 2013CB834704), the National Science Foundation of China (61275190 and 91233208), the Research Grants Council of Hong Kong (HKUST2/ CRF/10 and N_HKUST620/11), the Fundamental Research Funds for the Central Universities, the Program of Zhejiang Leading Team of Science and Technology Innovation (2010R50007), the Swedish VR Grant (621-2011-4620) and AOARD, and the Guangdong Innovative Research Team Program (201101C0105067115). We are grateful to Shuangshuang Liu and Xi Wang for their technical assistance with two-photon laser scanning microscopy and in vivo animal experiment, respectively.

\section{Author contributions}

D.W. and J.Q. designed the research. D.W., J.Q., W.Q. and A.Q. carried out the experiments. D.W., J.Q., B.Z.T. and S.H. analyzed data and participated in the discussion. D.W., J.Q., B.Z.T. and S.H. wrote and revised the paper. All authors reviewed the manuscript.

\section{Additional information}

Supplementary information accompanies this paper at http://www.nature.com/ scientificreports

Competing financial interests: The authors declare no competing financial interests.

How to cite this article: Wang, D. et al. Biocompatible and Photostable AIE Dots with Red Emission for In Vivo Two-Photon Bioimaging. Sci. Rep. 4, 4279; DOI:10.1038/srep04279 (2014).

cc)(1) This work is licensed under a Creative Commons Attribution-

By Na SA Nommercial-ShareAlike 3.0 Unported license. To view a copy of this license, visit http://creativecommons.org/licenses/by-nc-sa/3.0 